\title{
Auf das Thema „Sprache“ bezogene Witze im DaF-Unterricht und ein Versuch zu ihrer Didaktisierung mit Ausblick auf den interkulturellen Aspekt
}

\author{
Ebru Üstün (D), Nevşehir - Asuman Ağaçsapan (D), Eskişehir \\ https://doi.org/10.37583/diyalog. 1030835
}

\begin{abstract}
Deutsch)
Dieser Beitrag beschäftigt sich mit sprachbezogenen Witzen und ihrem konkreten Einsatz im Deutsch als Fremdsprachenunterricht. Der Fokus dieses Beitrags liegt auf der Empfehlung von Anwendungsbeispielen für die Nutzung von sprachbezogenen Witzen im DaF-Unterricht, die das vorrangige Ziel der Beschäftigung mit Stereotypen und der Entwicklung einer interkulturellen Kompetenz anstreben. Hierzu wird vorerst klargestellt was mit dem Begriff Witz gemeint wird, insbesondere, was als „sprachbezogener Witz“ in der Studie angesehen wird. Im Nachhinein wird auf die Bedeutung des Einsatzes von Witzen im Fremdsprachenunterricht eingegangen, sodass der Bedarf und Grund für die Anwendung dargelegt werden kann. Zur Lieferung adäquater Antworten auf die Forschungsfragen soll der Forschungsstand beschrieben werden und im praktischen Teil sollen Witze aus verschiedenen online Quellen ausgesucht und bewertet werden, die das Thema „Sprache“ vorweisen. Die Witze wurden zum Schluss als jeweils ein Didaktisierungsvorschlag erarbeitet. Mit den Didaktisierungsvorschlägen wurde aufgezeigt, dass sprachbezogene Witze sich für die Entwicklung einer interkulturellen Kompetenz, durch die Beschäftigung mit Stereotypen und Vorurteilen im DaF-Unterricht, sehr gut eignen können. Die Didaktisierungsvorschläge wurden nach dem Modell „Didaktische Analyse“ erstellt.
\end{abstract}

Schlüsselwörter: Sprachbezogene Witze, Deutsch Als Fremdsprache, Didaktisierung, Stereotype.

\section{Abstract (English) \\ Jokes related to the topic of "Language" in GFL lessons and an attempt to didacticize them with an outlook on the intercultural aspect}

This article deals with language-related jokes and their concrete use in German as a foreign language lesson. The focus of this article is on the recommendation of examples for the use of language-related jokes in GFL lessons, which strive for the primary goal of dealing with stereotypes and the development of intercultural competence. To this end, it is clarified what is meant by the term joke, in particular what is regarded as a "language-related joke" in the study. Afterwards, the importance of using jokes in foreign language teaching is discussed. In order to provide adequate answers to the research questions, the research status was analyzed and in the practical part, jokes were selected and analyzed from various online sources that show the topic of "language". At the end, these were each developed as a didactic suggestion. With the didactic suggestions it was shown that language-related jokes are very well suited for the development of intercultural competence by dealing with stereotypes and prejudices in GFL lessons. The didactic suggestions were created according to the "didactic analysis" model.

Keywords: Language-Related Jokes, German As A Foreign Language, Didactics, Stereotypes.

Einsendedatum: 27.09.2021

Freigabe zur Veröffentlichung: 01.12.2021 


\section{EXTENDED ABSTRACT}

Jokes mostly serve to loosen up the atmosphere, but the fact that, viewed in detail, they have even deeper backgrounds is only noticeable through intensive work with the topic. Jokes reflect the way of thinking of a certain culture, thus firstly, they offer the opportunity to gain an insight into the respective culture and secondly to recognize the perception of that society on the culture of the recipient himself. At best, this broadening of horizons leads to the development of intercultural competence. Intercultural competence plays an important role like never before, because both in the real and in the virtual world people meet each other more easily than ever before. Modern foreign language teaching has set itself the task of bringing the target language closer to the learner with all its aspects, including regional and cultural studies. Dealing with jokes in foreign language lessons creates a cultural image that cannot be found in this way in foreign language books. The focus of this work is on language-related jokes, in concrete terms this means that it is about the didacticization of jokes that focus on a language. This type of joke consequently includes evaluations about the respective community, which is thematized in the joke which attracts scholarly interest as Germany has been a country of migration for decades, which has led to a lot of jokes being formed in society about ethnic groups, as people often meet each other in everyday life. GFL lessons in particular offer the opportunity to work on these. The systematic structure of the jokes gives the teacher an overview of what to look out for during the lesson. Namely, jokes can be divided into lexical, phonological and syntactic jokes. Accordingly, the ambiguity in the joke can be attributed to the difference in meaning, in the sound of the words or in the sentence structure of the joke. Lexical jokes usually "only" require knowledge of the meanings of the respective word, while phonological jokes, for example, also require knowledge of pronunciation and intonation. Accordingly, it can be said that lexical jokes could have a priority in foreign language teaching and depending on the linguistic level of the learner, other types of jokes may be preferred.

The aim of this article is to recommend examples for the use of language-related jokes in GFL lessons. To this end, it is clarified what is meant by the term joke, what is regarded as a "language-related joke" in the study. Afterwards, the position of jokes in foreign language teaching is discussed. In the last part of the article, application examples for German lessons are presented with selected "language-related jokes". For this purpose, selected jokes are presented and an attempt is made to make them accessible for GFL lessons. For this purpose, jokes about other languages were presented with the aim of comparing the view of the respective culture in Germany and in one's own country. Every joke is first tried to explain, then ideas for didactics in GFL lessons with specific criteria for understanding the joke follow. Schu and Schu (1998) name "minimum goals" when using ethnic jokes in foreign language lessons. The treatment of such jokes should not be one-sided, but should be compared with one's own culture, so that learners do not misunderstand the target culture and view it as "carriers of stereotypes". It should be made clear that such images also exist in one's own culture and that they do not correspond to reality.

This structure is intended to try to provide answers to the following research questions with the work:

1. Is it possible to make students aware of prejudices and stereotypes in GFL lessons?

2. What requirements must be met when using "language-related jokes"?

3. How can "language-related jokes" be used and didactic in GFL lessons? 
The state of research was analyzed in order to provide adequate answers to the research questions mentioned. In the practical part, jokes were selected and analyzed from various Internet sources that dealt with the topic of "language". At the end, these were each developed as a didactic suggestion. All in all, the attempt was made to show that language-related jokes are a very suitable source for intercultural foreign language teaching. 


\section{Einleitung}

Gefühle spielen bekanntlich eine besondere Rolle im Prozess des Lernens, Behaltens und Erinnerns. Gaudo und Kaiser (2018) gehen in ihrer Arbeit auf die Wichtigkeit des Lachens beim Lernen ein und beschreiben, dass viele Studien den Einsatz von Humor befürworten und dass er zu einem besseren Lernerfolg führt. Die Funktionen von Humor im Unterricht allgemein, also nicht nur auf den Fremdsprachenunterricht bezogen werden wie folgt angegeben: „-Humor fördert das Lernen; - Humor weckt das Interesse der Schülerinnen und Schüler; - Lernstoff wird durch positive Emotionen und Humor besser behalten [...]; - Humor ist unser Bildungsauftrag" (Gaudo / Kaiser 2018: 19). Dabei wird Humor als „Lernbeschleuniger“ beschrieben. Viele Arbeiten bestätigen, die Annahme, dass das Lernen durch Humor, also Emotionen, erleichtert wird (vgl. Hascher / Edlinger 2008; Herrmann / Fiebach 2007; Vester 2001). Durch Gefühle werden demnach bestimmte Bereiche des Gehirns aktiviert, die das Lernen begünstigen (vgl. Gaudo / Kaiser 2018: 16; Mayer / Brückner 2011). Ein weiterer, zu beachtender Aspekt in Bezug auf Humor im Unterricht ist auch, dass Humor nicht zum Selbstzweck im Unterricht dienen sollte, sondern für einen Lerninhalt als Mittel eingesetzt werden sollte. Humoristische Inhalte wecken Assoziationen, die sich ebenfalls positiv auf den Erinnerungsprozess im Gehirn auswirken (vgl. Gaudo / Kaiser 2018: 31).

Alle Menschen sowohl Kinder als auch Erwachsene verfügen über einen Sinn für Humor, auch wenn dieser unterschiedlich ausfällt. Witze verbinden Menschen auf der Gefühlsebene, weil fast alle Kulturen den Witz kennen. Dieses verbindende Mittel bietet mit Sicherheit eine gute Möglichkeit zur Kulturvermittlung, zur interkulturellen Arbeit im Fremdsprachenklassenraum.

Ziel dieses Artikels ist es, Beispiele für den Einsatz sprachbezogener Witze im DaF-Unterricht zu empfehlen. Dazu wird geklärt, was unter dem Begriff Witz zu verstehen ist, insbesondere was in der Studie als „sprachbezogener Witz“ gilt. Anschließend wird die Bedeutung von Witzen im Fremdsprachenunterricht diskutiert. Im Anschluss daran werden Anwendungsbeispiele für den Deutschunterricht mit ausgewählten „Sprachwitzen“ vorgestellt. Dazu werden ausgewählte Witze präsentiert und versucht, diese für den DaF-Unterricht zugänglich zu machen. Hierfür wurden Witze über andere Sprachen im Internet mit der Eingabe von verschiedenen Sprachen recherchiert, mit dem Ziel, den Blick auf die jeweilige Kultur in Deutschland und im eigenen Land zu vergleichen. Jeder Witz wird zunächst versucht erklärt zu werden, dann folgen didaktische Ideen für den DaF-Unterricht mit konkreten Kriterien zum Verständnis des Witzes. Schu / Schu (1998) nennen „Mindestziele“ bei der Verwendung ethnischer Witze im Fremdsprachenunterricht. Diese gelten auch für Nationenwitze. Der Umgang mit solchen Witzen sollte nicht einseitig erfolgen, sondern sollte mit der eigenen Kultur verglichen werden, damit Lernende die Zielkultur nicht missverstehen und als „Stereotypträger" betrachten. Es sollte klargestellt werden, dass solche Bilder auch in der eigenen Kultur existieren und nicht der Realität entsprechen müssen.

Die Arbeit soll dazu dienen Antworten auf folgende Forschungsfragen zu geben: 
1. Kann man Lerner im DaF-Unterricht für Vorurteile und Stereotype sensibilisieren?

2. Welche Voraussetzungen müssen bei der Verwendung von „sprachbezogenen Witzen“ erfüllt sein?

3. Wie können „sprachbezogene Witze“ im DaF-Unterricht didaktisch eingesetzt werden?

Durch eine deskriptive Beschreibung und eine neutrale Bewertung der Witze wurde versucht adäquate Antworten auf die genannten Forschungsfragen zu geben. Insgesamt wurde aufgezeigt, dass sprachbezogene Witze eine sehr geeignete Quelle für den interkulturellen Fremdsprachenunterricht sind.

\section{Der Begriff Witz und seine Merkmale}

Witze spielen eine Hauptrolle dabei, wenn von Humor die Rede ist. In der Humortheorie werden vorwiegend drei Erklärungsmodelle angewendet, die nach Kotthoff dargelegt werden sollen. Sie bieten der Lehrkraft ein Verständnis dafür, was Witze enthalten und wie sie beim Lernenden ankommen können. Die drei Modelle Inkongruenz, Degradation und Entspannung stehen zueinander in Verbindung und „verstärken sich gegenseitig oder schwächen sich ab“ (Kotthoff 2006: 9). Unumstritten in der Humortheorie ist, dass Inkongruenz ein essentielles Element für das Komische darstellt. Nach Kothoff (2006) sahen Freud und Schopenhauer die Inkongruenz als Grundlage des Humors. Dieses setzt also das Verstehen einer NichtÜbereinstimmigkeit im Inhalt des Witzes voraus. Degradation und Aggression sind weitere Modelle, welche ihren Ursprung bei Platon haben (vgl. Kotthoff 2006: 12). Hier wird beschrieben, dass Lachen aus einem Überlegenheitsgefühl dem Gegenüber entspringt. Dem Witz wird ein aggressiver Charakter zugeschrieben, da er ,zwei potentielle Opfer" hat: der Inhalt oder das Ziel des Witzes und der Rezipient des Witzes, von dem erwartet wird den Witz zu verstehen. Dieses spielt wiederum auf den Aspekt des Stereotypen in Witzen an. Das Überlegenheitsgefühl und somit die Degradation wird nicht als Voraussetzung angesehen, jedoch können humoristische Elemente diese Eigenschaft beinhalten (vgl. Kotthoff 2006: 12). Kotthoff warnt, dass die humoristische Kommunikation nicht immer harmlos sei. „Sie kann Spannung abführen oder aufbauen, Feindschaft oder Freundschaft ausdrücken, individuellen oder gemeinsamen Interessen dienen. Sie kann im Dienste von Subversion stehen, aber auch im Sinne der Bestätigung von Herrschaft" (Kotthoff 2006: 12). Diese Eigenschaften sollten mit Sicherheit auch Lehrkräfte vor Augen haben, wenn sie Lernende mit solchen Texten konfrontieren. Das Hauptanliegen eines Unterrichts mit Witzen könnte deshalb eher auf die Bewusstmachung von Spannungen, Feind- oder Freundschaften und gemeinsamen Interessen liegen. Dieses würde mit Sicherheit im Interesse des interkulturellen Ansatzes liegen. Das dritte Modell, also die Entspannung, beschreibt das, was dem Laien als Erstes in den Sinn kommt, wenn es um Humor geht (vgl. Kotthoff 2006: 12). Wie unschwer am Namen zu erkennen ist, geht es hierbei darum Spannungen 
abzubauen. Dieser Aspekt spielt mit Sicherheit auch eine Rolle im Unterricht, da bekanntlich Stress das Lernen in größter Weise hemmt. Deshalb ist eine lockere Atmosphäre von Vorteil. Nach der Einführung in die Humortheorie, die ebenfalls Anhaltspunkte für den Einsatz von Witzen im Fremdsprachenunterricht enthält, soll nun konkreter auf den Begriff Witz eingegangen werden.

Der Begriff Witz liegt als Textform neben anderen sozialwissenschaftlichen Forschungsfeldern im Interesse der linguistischen Forschung. Einige Definitionen werden im Folgenden angegeben, sodass eine Orientierung geschaffen werden kann. Im Duden wird der Witz folgendermaßen definiert: „,[prägnant formulierte] kurze Geschichte, die mit einer unerwarteten Wendung, einem überraschenden Effekt, einer Pointe am Ende zum Lachen reizt [...]“ (Dudenredaktion, o.J.). Diese Definition enthält die meisten Stichpunkte, auf die in den folgenden Definitionen im Detail angegangen werden soll. Diese Stichpunkte sind unter Anderem „unerwartete Wendung“ und „Pointe“, die in keiner Definition zu fehlen scheint. Mit Pointe meint man genau die Stelle im Witz, in der die unerwartete Wendung, die Überraschung stattfindet. Der Witz hat also als eine Funktion auch den Rezipienten zum Lachen anzuregen, er ist also ein Mittel des Humors. Witze dienen oft zur humorvollen Darstellung von Themen oder Situationen. Der komische Effekt im Witz wird meist über die Mehrdeutigkeit von Worten erschaffen. Die beabsichtigte Bedeutung eines Wortes kann also missverstanden werden, sodass dieser Irrtum zu einer witzigen Situation führt. Diese Eigenschaft von Wörtern wird als Polysemie bezeichnet und sie spielen eine wichtige Rolle in Witzen.

Nach Röhrich wird der Witz folgendermaßen definiert „Ein Witz ist eine kurze, Lachen erregende Erzählung, die in einer Pointe gipfelt" (Röhrich 1977; zitiert nach Szczęk 2006: 170). Auch in dieser Definition steht die Pointe im Vordergrund, da der Witz hier ,gipfelt““. „Der Witz kann als selbständiger, fiktionaler, komisch pointierter Prosatext in mündlicher und/ oder schriftlicher Form angesehen werden, dessen besonderes Merkmal die komprimierte, intellektuell zugespitzte sprachliche Form ist" (Middeke / Murdsheva 2008: 224). Middeke und Murdsheva (2008) unterstreichen die mündliche und schriftliche Form des Witzes und nennen das Wort ,intellektuell“, was uns zum Thema Verstehen eines Witzes bringt. Unumstritten ist, dass das Verstehen eines Witzes bestimmte Voraussetzungen verlangt. Eine dieser Voraussetzungen ist natürlich erstens, wenn es um Witze in einer Fremdsprache geht, die sprachliche Voraussetzung und zweitens ein kulturelles Vorwissen. Middeke und Murdsheva (2008: 224) erweitern dieses mit dem Begriff der „Korrespondenzbereichtschaft“ des Hörers nach Schubert und Lixfeld

Das Verstehen von Witzen setzt aber nicht nur „kulturspezifische[s] Vorwissen“ (Schubert: 137), sondern auch entsprechende Wertungen beim Hörer/ Leser voraus und verlangt ihm eine mehr oder weniger große emotive Korrespondenzbereitschaft ab. „So liegt es nahe“, meint deshalb Lixfeld (1978a: 18), den Witz als Träger von Informationen und Intentionen im Kommunikationsprozess, als Denknorm und Verhaltensmuster schaffendes oder zumindest verfestigendes oder jedoch als kritisierendes und in Frage stellendes Medium der Bewußtseinsbildung zu betrachten. (Middeke / Murdsheva 2008: 224) 
Demnach muss der Hörer dazu bereit sein, einen Witz im Inhalt zu erkennen und entsprechende Wertungen, die Witze enthalten können, als Witz zu verstehen. Dieses ist auch beim Einsatz im Fremdsprachenunterricht zu beachten, da sich Witze häufig an Stereotype bedienen und somit häufig emotionale Reaktionen auslösen können. Doch darauf soll später eingegangen werden.

Ueda beschreibt den Witz folgenderweise:

\begin{abstract}
Witztexte und Karikaturen geben Anlass zum Nachdenken und zu Kritik. Witztexte und Karikaturen erlauben es dem Leser häufig zu einer neuen Erkenntnis zu kommen und die Dinge anders zu sehen. Wir sind nicht nur zum Zweck des Lachens da. Sie stellen auch eine Erkenntnis oder Anschauungsweise da. Manchmal braucht man die Dinge nur anders $\mathrm{zu}$ sehen um sich von einer festgefahrenen Betrachtungsweise befreien $\mathrm{zu}$ können. (2013: 2)
\end{abstract}

Außer der Funktion des Humors regen Witze also zum Nachdenken an, noch wichtiger bieten sie eine Einsicht in eine bestimmte Lebenswelt. Dieses liegt im Interesse dieser Arbeit, da man mit dem Einsatz von Witzen auch das Ziel anstrebt, interkulturelles Wissen voranzutreiben.

Middeke und Murdsheva (2008) unterstreichen die Aussage Uedas damit, dass sie Witze als Spiegel der Gesellschaft beschreiben. Mit den Stereotypen in solchen Witzen kann ein Kennenlernen der Zielkultur auf eine besondere Art und Weise mit Humor ermöglicht werden, da Witze ein anderes Bild der jeweiligen Gesellschaft wiederspiegeln. Andererseits kann aber auch die Sicht auf das Eigene betrachtet werden, was eigentlich das Grundlegende der interkulturellen Didaktik darstellt.

\title{
2.1 Sprachbezogene Witze und Stereotype
}

Unter sprachbezogenen Witzen verstehen wir im Rahmen dieser Arbeit solche Witze, in denen die Sprache, sei es die deutsche Sprache oder eine andere, im Mittelpunkt steht. Der Witz muss also die Sprache als Gegenstand haben. Meistens entstehen die komischen Situationen in diesen Witzen durch ein Missverständnis, das auf eine Sprachbarriere zurückzuführen ist. Zu den genannten Witzen gehören auch meistens die sogenannten Ethno-Nationenwitze, da die beiden Phänomene Sprache und Nation/ Ethnie eng in Verbindung zueinanderstehen. Als ethnische Witze werden einerseits solche Witze bezeichnet, die von ethnischen Gruppen über andere ethnische Gruppen erzählt werden und andererseits auch solche Witze, die eine ethnische Gruppe über sich selbst erzählt (vgl. Szczęk 2006: 171). Für Ethnie wird im Wahrig „Stamm, Volk od. Volksgruppe“ angegeben, ethnisch wiederum wird als ,zur Ethnologie gehörig, auf ihr beruhend [...] Eine Ethnie, Volksgruppe betreffend" definiert" (Wahrig-Burfeind 2011: 480). Ein weiterer Begriff, der für diese Arbeit in Frage kommt ist die Nation oder national. Nation wird im Wahrig als „Nach Abstammung, Sprache und Sitte, kultureller u. politischer Entwicklung zusammengehörige, innerhalb der gleichen Staatsgrenzen lebende, politische Gemeinschaft" bezeichnet (Wahrig-Burfeind 2011: 1055). Beide Aspekte werden in dieser Arbeit angesprochen. 
Als Nationen-und Ethnowitze werden sowohl Witze von als auch über Nationen und ethnische(n) Gruppen bezeichnet. Die Zielgruppe wird durch die Nation, die Religion, die Ethnie oder regionale Zugehörigkeit bestimmt. Nationen-und Ethnowitze beruhen auf Klischeevorstellungen und weisen eine mehr oder weniger große Anzahl an Stereotypen auf. (Middeke / Murdsheva 2008: 223)

Bei der Betrachtung der Witze, die für diese Studie ausgewählt wurden, fällt auf, dass sprachbezogene Witze, Stereotype beinhalten. Stereotype werden als „Bilder im Kopf“ (Lippmann 1922, zitiert nach Szczek 2006: 170) definiert, die verallgemeinert und auf eine Gruppe zugeschrieben werden. Diese Gruppe kann jeder Art sein, es können Stereotype über bestimmte Berufsgruppen, nationale oder ethnische Gruppen oder auch Geschlechter sein. Durch Stereotype versucht man Unbekanntem ein Gesicht zu verleihen. Stereotype werden innerhalb einer Kultur von Generation zu Generation weitergeleitet (vgl. Szczęk 2006: 170). Schu / Schu (1998) definieren den Begriff des ethnischen Stereotyps mit dem Begriff des sozialen Stereotyps. Dieses wiederum beinhaltet das sogenannte subjektive Wissen, welches typische Verhaltensweisen, Eigenschaften und Handlungen von Mitgliedern der eigenen oder einer fremden Gruppe sind. Es ist eine subjektive Wahrnehmung und Betrachtungsweise.

Wenn beispielsweise die türkische Sprache in einem Witz zum Gegenstand gemacht wird, dann sind auch oft die Türken als Protagonisten in diesen Witzen vorhanden, dies gilt mit Sicherheit für alle Sprachen, Nationen und Ethnien. Das bedeutet ebenfalls, dass sprachbezogene Witze Stereotype enthalten können. Die Arbeit mit Stereotypen im Fremdsprachenunterricht sollte nicht vermieden werden, da sie bedeutend für das interkulturelle Bewusstsein ist.

\subsection{Klassifizierung von Sprachwitzen nach Lew}

Dass Witze als Gegenstand vieler linguistischer Forschungen auf verschiedene Weisen untersucht werden, wurde schon vorher angesprochen. Demnach gibt es auch zahlreiche Ansätze zur Kategorisierung von Witzen. Die Suche im Internet nach Witzen, stellt primär eine Darstellung nach Themenfeldern dar. Man kann also sogenannte Schülerwitze, Lehrerwitze, Frauenwitze, Coronawitze, Bayernwitze, Türkenwitze usw. auffinden. Da wir uns im Rahmen dieser Arbeit mit „sprachbezogenen Witzen“ beschäftigen, scheint die Klassifizierung nach Lew (zitiert nach Semiz 2014) angemessen, da dies zum besseren Verstehen der Witze beitragen kann. Nach Semiz (2014) unterscheidet Lew drei Arten von Sprachwitzen: lexikalische, phonologische und syntaktische Witze. Demnach kann die Mehrdeutigkeit im Witz auf die Unterschiedlichkeit in der Bedeutung, im Klang der Wörter oder in der Satzstruktur des Witzes zurückzuführen sein. „Der auf der Mehrdeutigkeit des Wortes basierende Witz kann auch polysemer Witz heißen und seit langer Zeit galt er als die typischste Form des Worthumors“ (Hoffmann 2010: 79). Ein Wort also, dem mehrere Bedeutungen zugeschrieben werden, kann zu einem komischen Effekt im Witz führen. Diese Art Witze zählen zur Kategorie der lexikalischen Witze: „Zahnarzt zum Patienten: ,Sie brauchen eine Krone'. 
-Patient: ,Endlich versteht mich jemand““”(http-1).

Der komische Effekt in diesem Witz basiert darauf, dass das Wort „Krone“ in diesem Kontext, nämlich der der Zahnmedizin, vom Patienten als der Kopfschmuck eines Königs oder einer Königin aufgefasst wird. Der Arzt spricht jedoch von einer Zahnkrone, die zur Behandlung eines Zahnproblems nötig ist.

Der phonologische Witz nach Lew beschreibt einen Effekt, der dadurch entsteht, dass durch die Aussprache eines Tones im Wort oder im Satz ein Missverständnis entsteht. Diese können bei ähnlicher Aussprache auch mehrere Bedeutungen hervorrufen:

„- Was heißt Ofen auf Chinesisch?

- Hei Zung.“, (http-2).

oder:

„Woraus machen die Deutschen ihre Autos? - Aus Kruppstahl. Woraus machen die Schweden ihre Autos? - Aus Schwedenstahl. Und woraus machen die Polen ihre Autos? - Aus Diebstahl.“ (http-3).

Beim ersten Witz ist eine Anspielung auf den chinesischen Klang wiederzufinden. Für Sprecher des Deutschen klingt chinesisch etwas „,abgehackt“, deshalb die Trennung zwischen den Silben des Wortes „Heizung“. Natürlich spielt das Wort „Heizung“ selbst auch eine Rolle im Witz, da es wie der Ofen, zum Heizen genutzt wird. Durch die Aussprache wird dieser Witz als komisch empfunden. Beim zweiten Witz geht es um Aussprache des Wortes „Stahl“ im Sinne von Eisen als Werkstoff zur Autoherstellung in Deutschland oder Schweden und des Wortteils ,-stahl“ im Wort Diebstahl, was auf die Polen bezogen wird. Dieses bezieht sich auf das Stereotyp über die Polen.

Die dritte Kategorie, der syntaktische Witz, entsteht durch die Wortstellung im Satz:

„-Lehrerin: Was hast du da im Mund?

- Lukas: Ein Kaugummi. -

-Lehrerin: Sofort in den Papierkorb!

- Lukas: Auch das Kaugummi?““(http-4).

Dieser Witz basiert auf der Aussage der Lehrerin, dass Lukas seinen Kaugummi in den Papierkorb schmeißen soll. Sie drückt den Satz aber unvollständig aus, wodurch der lustige Effekt entsteht, dass Lukas sie missversteht. Durch das bewusste Auswählen eines Witzes, kann man den Fokus der Vorbereitung für einen Fremdsprachenunterricht erleichtern. So ist man in der Lage, gezielte Vorentlastungen für die Lernenden anzubieten. 


\section{Zum Einsatz von Witzen im FSU}

Besonders der ethnische Witz wird von Schu / Schu (1998) als relevant für den Fremdsprachenunterricht angesehen, da er ein landeskundlicher Gegenstand ist. Es befürworten weitere Arbeiten den Einsatz von Witzen als authentisches Material im Fremdsprachenunterricht (vgl. Ağaçsapan u.a. 2018; Hargaßner 2018; Utri 2012; Voltrová 2018). In ihrer Arbeit betonen Schu / Schu immer wieder den Aspekt des Zusammenhangs von Landeskunde und ethnischen Witzen und schreiben, dass Witze Horizonte erweitern können, dadurch, dass sie zur Meinungsbildung beitragen, zum interkulturellen Vergleich und zur selbständigen Weiterarbeit anregen (Schu / Schu 1998: 138). Witze dienen demnach als Kulturträger und können das Interesse zur Weiterarbeit von Lernenden wecken. Vor allem machen Schu / Schu (1998) darauf aufmerksam, dass Witze eine andere Art von Landeskunde wiedergeben, die in Lehrwerken nicht zu finden sind. Sie präsentieren keine heile Welt ohne Probleme, ethnische Witze und Nationenwitze können deshalb $\mathrm{zu}$ einem anderen, neuen Blickwinkel führen.

„Der Hauptakteur des heutigen Kommunikationsnetzes, in dem, dank sozialen Medien, jeder jeden auf einfachste Weise erreichen kann, ist zweifelsohne die

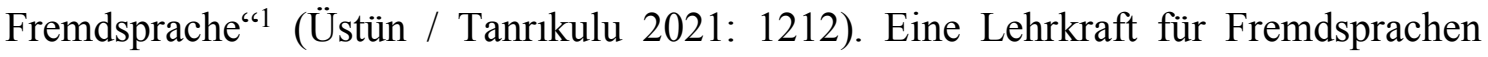
sollte sich demnach nicht die Möglichkeit entgehen lassen, im Unterricht kulturelle Elemente mit Witzen darzubieten. Die interkulturelle Kompetenz ist heute eine Anforderung des modernen Fremdsprachenunterrichts. Hierfür steht der interkulturelle Ansatz in der Fremdsprachendidaktik. Huneke und Steinig (2000) halten hierzu fest
Im Fremdsprachenunterricht geht es jetzt nämlich um die Fähigkeit zum Fremdverstehen im umfassenden Sinn. Dies setzt, integriert in die sprachlichen Kompetenzen, vor allem auch Einsichten in die Kulturbedingtheit kommunikativen Handelns in der Zielsprache und in der Ausgangssprache voraus. Dabei können auch Einstellungen zu Normen der Herkunftskultur modifiziert und so Beiträge zur Entwicklung der eigenen Identität geleistet werden. (2000: 161)

Diese Kompetenz beschreibt nicht nur das Kennenlernen der Zielkultur, sondern zielt darauf $a b$, durch das Kennenlernen ein Bewusstsein zu erlangen und einen anderen Blickwinkel für die eigene Kultur zu gewinnen. „Vielmehr geht es um das Erreichen dessen, was der Interkulturalist Bennet als den ,dritten Ort" bezeichnet hat, der aber auch ein vierter und fünfter sein kann. Es geht also um das Anstreben von Perspektiven, die miteinander ausgeglichen, aber auch kontrovers, koexistieren können" (Roche 2005: 160f.). Es ist also eine Entwicklung, die Lernende durchmachen. Roche (2005) vergleicht dieses mit der Mehrsprachigkeit, so wie man sich in mehreren Sprachen auszudrücken weiß, so sollte man auch durch interkulturelle Kompetenz sich in mehreren Kulturen ,zu Hause“ fühlen. Um diese Perspektiven zu erlangen sollte man mit Sicherheit in der Lage sein das Eigene aus der Sicht des Anderen zu sehen. Zur Verschaffung eines Bildes darüber, wie man selbst aus der fremden Perspektive wahrgenommen wird, dienen Witze ebenfalls sehr gut. Schließlich zeigen insbesondere ethnische Witze und Nationenwitze das, was die jeweilige Kultur über die eigene Kultur

\footnotetext{
${ }^{1}$ [Übersetzung der ersten Verfasserin]
} 
denkt. Die Aufgabe der Lehrkraft hierbei ist es, den Lernenden bewusst zu machen, dass diese Bilder von Vorteilen und Stereotypen geprägt sind. Hierzu schreiben Middeke und Murdsheva folgendermaßen:

Durch die Beschäftigung mit den Ethnostereotypen in Witzen sollen die Lerner sich
ihrer ambivalenten, einerseits verstehensfundierenden und andererseits
verstehenshemmenden Funktion bewusst werden, sie sollen Wahrnehmungsfertigkeiten
erwerben, mit denen sie sich der Existenz und den Funktionen von Stereotypen bewusst
werden, und kognitive Fähigkeiten, mit denen sie diese hinterfragen und auch
widerlegen können. (Middeke / Murdsheva 2008: 224)

Das Wissen über die Zielkultur wird als Voraussetzung für die pragmatische Nutzung der gelernten Sprache angesehen. Kulturwissen und interkulturelle Kompetenz werden als Voraussetzung zur Kommunikation beschrieben. Diese Kommunikation ist heute von großer Bedeutung, da viele Lerner des Deutschen als Fremdsprache Deutschland, vor allem in der Türkei, als ein attraktives Land ansehen und zukunftsorientiert ein Leben in Deutschland vorstellen. Oft ist dieses sogar die Motivation der Lernenden (Auswärtiges Amt 2020). Um Lernenden diese Fähigkeit zu vermitteln, bedarf es als Voraussetzung das Vorhandensein der interkulturellen Kompetenz bei Lehrkräften, diese sollten sich als Vermittler zwischen den beiden Kulturen sehen und dementsprechend handeln, da sie für Lernende des Deutschen im Ausland die erste Anlaufstelle für die deutsche Sprache und Kultur sind.

\subsection{Kriterien bei der Auswahl von Witzen für den FSU}

Einen Witz zu verstehen, verlangt besondere Fertigkeiten, auch schon in der Muttersprache. Neben sprachlichen Kenntnissen sind meistens auch landeskundliche Kenntnisse von Nöten. Neben dem landeskundlichen Aspekt stellt sich auch die Frage der Themen, die angesprochen werden können/ dürfen oder nicht. „Es sollte hier nicht unterlassen werden, auch auf gewisse Gefahren hinzuweisen, die sich aus potentiellen "Grenzverletzungen « ergeben können. So muss beispielsweise (besonders) auf gewisse Tabu-Bereiche Rücksicht genommen werden" (Hohenhaus 2000: 445). Es ist also wichtig die Gruppe und die eigene Kultur zu kennen, sodass man keine verletzenden Texte auswählt, die die Lernenden kränken o.Ä. kann. „Neben generell geltenden TabuBereichen wie Tod, Sex, Religion, etc. können solche Grenzen auch interkultureller Natur sein" (Hohenhaus 2000: 434). Falls die jeweiligen Kulturen einen gemeinsamen Schnittpunkt im geschichtlichen Kontext haben, sollten diese Themen, wenn überhaupt, mit besonderer Vorsicht angegangen werden. Hier ist das Feingefühl der Lehrkraft gefragt. Somit eignen sich nicht alle Witze zur Verwendung im Unterricht. Diese werden unter dem Kriterium der Angemessenheit zusammengefasst. Löschmann beschreibt diesen Begriff mit folgenden Punkten ,1) Alter der Lernenden 2) Interessen 3) Weltwissen 4) Kompetenzstand in der Zielsprache 5) Erfahrungshorizont 6) kulturspezifische Verstehensvoraussetzungen und nicht zuletzt 7) nationales, moralisches, religiöses Empfinden“ (Löschmann 2015: 37). Das Verstehen eines Witzes, vor allem eines, das Stereotype beinhaltet, und die Bemühung der Lehrkraft ein Bewusstsein hierfür zu schaffen bedürfen mit Sicherheit einer bestimmten kognitiven 
Reife. Als zweites Kriterium nennt Löschmann (2015) den Bezug zum Lern- und Lehrstoff, welches auch schon unter dem Aspekt des „Lachens und Lernens“ angesprochen wurde.

Die Kategorien nach Lew und die Arbeit Semiz (2014) können uns eine Orientierung darüber geben, welche Art von Witzen für Fremdsprachenlerner geeignet ist. In ihrer Arbeit über das Verstehen von Witzen der Englischlerner in der Türkei fand sie heraus, dass die Teilnehmenden lexikalische Witze leichter verstanden als phonologische oder syntaktische Witze. Dieses Ergebnis scheint nachvollziehbar, wenn man bedenkt, dass jede Art von Witzen eine andere Fertigkeit abverlangt. Lexikalische Witze verlangen meistens „nur“ die Kenntnis der Bedeutungen des jeweiligen Wortes, während beispielsweise phonologische Witze zu dem auch Kenntnis zur Aussprache und Betonung verlangen. Demnach kann man sagen, dass lexikalische Witze einen Vorrang im Fremdsprachenunterricht haben könnten, je nach dem sprachlichen Niveau der Lernenden kann man andere Arten von Witzen vornehmen.

In ihrer Arbeit geht auch Çakır (2006) auf die Kriterien bei authentischen Materialien für den Fremdsprachenunterricht ein. Sie beschreibt zwar hauptsächlich Kriterien für Lehrwerkstexte, dennoch scheinen einige ihrer Kriterien auch angemessen und beachtenswert für die Textsorte Witze als authentische Materialien zu sein. Als eines der Kriterien beschreibt Çakır die mediale Präsentation des Materials, also auf welche Art und Weise die Lernenden mit dem Text konfrontiert werden. Der Witz ist im realen Leben meistens eine Textsorte, die mündlich vorzufinden ist. Çakır (2006) betont, dass im Unterricht multisensorisch gearbeitet werden sollte, sodass das Behalten gestärkt werden kann. Demnach sollten die mündlich existierenden Witztexte mit Bildern, schriftlichen Texten oder Rollenspielen unterstützt werden. Die mediale Präsentation ist in Verbindung mit dem Kriterium der äußeren Gestalt des Textes. In Witzen gibt es häufig keine äußeren Erkennungsmerkmale wie Überschriften oder Bilder, dennoch können diese als Verstehenshilfe hinzugefügt werden. Das Kriterium der ,sprachlichen Einkleidung“" beschreibt Çakır (2006) als die sprachliche Vielfalt in Texten, ob es zum Beispiel Jugendsprache oder Dialekte beinhaltet. Außerdem werden Kriterien für den syntaktischen, lexikalischen, phonetischen und diskursiven Bereich vorgestellt, auf die im Didaktisierungsteil der Arbeit eingegangen werden sollen. Ein anderes Kriterium Çakırs (2006) ist die „Intention des Textes“ in der realen Welt, die im Unterricht nicht verändert werden sollte. Witze bleiben also immer noch Witze und sollten im Unterricht auch als solche vorgestellt werden. Das letzte Kriterium in der Arbeit Çakırs (2006) sind die „Adressaten der Texte“, wobei die eigentlichen Adressaten $\mathrm{zu}$ erkennen sein sollten und die Adressaten im Unterricht eine Rolle spielen. Texte sollen also einen Bezug zum Adressaten im Unterricht aufweisen und an „Erfahrungen und Vorwissen“ anknüpfen. 


\subsection{Sprachbezogene Witze und Ideen zu ihrer Didaktisierung}

Als primäres Ziel beim Einsatz von „sprachbezogenen“ Witzen im Fremdsprachenunterricht wird in dieser Arbeit die interkulturelle Kompetenz und damit verbunden die Arbeit mit Stereotypen angesehen, wobei auch sprachliche Aspekte wie die der Grammatik oder des Wortschatzes als Ziel gesetzt werden können. „Als Einstieg in die Stereotypenproblematik kann die Beschäftigung mit der Fremdwahrnehmung der eigenen Kultur oder der Zielkultur gewählt werden“" (Middeke / Murdsheva 2008: 224). „Wie werde ich in einer anderen Kultur angesehen?", diese Frage kann also eine Einstiegsfrage zur Beschäftigung mit sprachbezogenen Witzen dienen. Hierzu werden im Folgenden ausgewählte Witze präsentiert und versucht für den DaF-Unterricht zugänglich zu machen. Im Weiteren werden Witze über andere Sprachen mit dem Ziel zum Vergleich der Betrachtung der jeweiligen Kultur in Deutschland und im eigenen Land dargestellt. Jeder Witz wird vorerst versucht zu erklären, dann folgen Ideen zur Didaktisierung im DaF-Unterricht mit spezifischen Kriterien für das Verstehen des Witzes. Auch Schu / Schu (1998) nennen „Minimalziele“ beim Einsatz von ethnischen Witzen im Fremdsprachenunterricht. Die Behandlung solcher Witze soll nicht einseitig, sondern mit der eigenen Kultur im Vergleich geschehen, sodass Lernende die Zielkultur nicht missverstehen und sie als „Träger von Stereotypen“ betrachten. Klargestellt soll unbedingt werden, dass solche Bilder auch in der eigenen Kultur existieren und dass diese nicht der Realität entsprechen müssen.

Der folgende Teil der Arbeit beschäftigt sich mit der Frage, wie man sprachbezogene Witze im DaF-Unterricht konkret einsetzen kann. Unter den auserwählten Witzen gibt es einige, die sich mit der türkischen Sprache und Kultur beschäftigen und andere, bei denen es um andere Sprachen geht. Wieder einige dieser Witze erfordern eine tiefere Vorarbeit der Lehrkraft, da beispielsweise historische Hintergründe oder weiteres Weltwissen zum Verstehen des Witzes erforderlich sind. Durch die Kategorisierung nach Lew kann eine Idee darüber entstehen, ob dieser Witz im Fremdsprachenunterricht eher leichter oder weniger leichter zu verstehen ist. Bei den Vorschlägen wird davon ausgegangen, dass die Lehrkraft über das notwendige sprachliche und landeskundliche Wissen zum Verstehen der Witze verfügt.

Bei den Didaktisierungen der Witze wurde das Kriterium der Angemessenheit in Bezug auf Weltwissen, Interessen, Kompetenzstand in der Zielsprache, Erfahrungshorizont, kulturspezifische Verstehensvoraussetzungen und nationales, moralisches, religiöses Empfinden nach Löschmann (2015) versucht einzuhalten, demnach wurden vorentlastende Ideen vorgeschlagen. Die Didaktisierungsvorschläge wurden nach dem Modell „Didaktische Analyse als Kern der Unterrichtsvorbereitung“, das nach Klafki (zitiert nach Bimmel 2011) erstellt.

Das Modell, dass vielfach variiert und adaptiert wurde, stellt und beantwortet folgende Fragen: - Welche Bedeutung hat das Thema für die Gegenwart, welche für die Zukunft? ; - Welche konkreten Aufgaben lassen sich daraus ableiten und welche Schritte können zu ihrer Lösung beitragen? ; - Inwiefern hat das Angebot exemplarische Bedeutung, bezieht es sich also auf etwas Allgemeines? ; - Wie kann das Angebot für die Schüler nachvollziehbar, zugänglich angeboten werden? (Bimmel et al. 2011:40) 
Dieses Modell dient dazu, dass Lehrende ihren Unterricht strukturiert planen und dass Lernende sich dem zu behandelnden Text schrittweise nähern. Für jeden Vorschlag wird versucht auf die gegenwarts- und zukunftsbezogene Perspektive einzugehen, auf die exemplarische Bedeutung und der Struktur und Zugänglichkeit für die Lernenden. Wobei der zukünftliche Bezug für den DaF-Unterricht in unseren Fällen die interkulturelle Kompetenz als Hauptziel genannt werden kann.

\subsubsection{Witz 1: Dialekte des Deutschen, Wahrnehmung des österreichischen Dialekts}

„Ein Österreicher bei McDonald's: „I hätt gern a Hendl!“ Der Angestellte: „Äh, Sie meinen Chicken?“ Darauf der Österreicher: „Nein, ned schicken! Ich ess“ es gleich hier" (http-5).

Das erste Beispiel ist ein typischer Österreicher-Witz, bei dem das Thema, die Abweichung des österreichischen Deutschs zum Standarddeutschen ist. Dieses Thema findet meist seinen Platz in Lehrbüchern, deshalb kann es sein, dass Lernende eine Idee darüber haben, dass es hier Unterschiede geben kann. Die Bestellung „Hendl“ von einem Österreicher im McDonalds wird durch den Angestellten als „Chicken“ verbessert, da „Hendl“ in Österreich für „Hähnchen“ benutzt wird, und das „Hähnchen“ im McDonalds als „Chicken“ angeboten wird. Was hier zum komischen Effekt führt ist, dass der Österreicher das englische Wort „Chicken“ mit dem deutschen Wort „schicken“ verwechselt. Dieses Missverständnis geschieht dadurch, dass die beiden Wörter einen ähnlichen Klang haben. Somit ist dieser Witz als ein phonetischer Witz nach Lew zu kategorisieren.

Für den Unterricht eignet es sich, mit einem McDonalds-Logo zu beginnen, jeder Lernende auf der Welt erkennt dieses Logo wieder. Eine Einstiegsfrage kann lauten: „Was bestellen Sie sich am liebsten bei McDonalds?“. Es kann ein Dialog zwischen einem McDonalds-Mitarbeiter und einem Kunden erstellt werden, sodass eine Einführung zum Thema entsteht und Vorwissen aktiviert wird. Nach dem Präsentieren der von den Lernenden erstellten Dialoge als Rollenspiel kann nun der Witz präsentiert werden. Diese Stufe kann als Gegenwartsbezug, denn es geht um einen Einstieg mit einem Thema, das fast jedem bekannt ist. Von diesem Schritt aus wird ebenfalls die Zugänglichkeit für Lernende erklärt. Diese Frage soll das „Wie“ im Unterricht beantworten.

Das Vorlesen sollte einem Lernenden überlassen werden, sodass der erste Überraschungseffekt eintreten kann. Denn wahrscheinlich wird das österreichische Deutsch sie verwirren. Vielleicht werden sie versuchen das „I“ am Anfang wie das „I“ im Englischen betonen. Nach einigen Versuchen kann die Lehrkraft den Witz vorlesen und darauf aufmerksam machen, dass dieses ein Dialekt des Deutschen ist. Nun kann die Frage folgen „Was bestellt der Österreicher?“. Hierauf kann eine Gruppenarbeit folgen, in der versucht wird, den Dialog zu entschlüsseln mit der Information, dass „Hendl“ für „Hähnchen“ steht. Das Thema „Konjunktiv II“ zu kennen erleichtert hier das Vorgehen. 
Nach einigen Versuchen kann die Lehrkraft den Satz auf Standarddeutsch festhalten „Ich hätte gern ein Hähnchen“ und „Nein, nicht schicken, ich esse gleich hier“. Nun folgt die Frage zur Pointe des Witzes „Warum hat der Österreicher den Angestellten falsch verstanden?“. Zur Hilfe können die Wörter „,chicken“ und „schicken“ an die Tafel geschrieben werden. Der Grund, dass diese Wörter sehr ähnlich klingen wird in Kürze bestimmt genannt. Die Erklärung kann auch in der Muttersprache gemacht werden. Die Behandlung dieses Witzes im Unterricht trägt weniger grammatische Ziele. Hier geht es in erster Linie darum, ein ,anderes“ Deutsch vorzustellen und was passieren kann, wenn das Standarddeutsch und eines seiner Dialekte aufeinandertreffen.

Als Ergänzung können auch aus dem Internet Videos vorgespielt werden, in denen der österreichische Dialekt vorkommt. Somit wird den Lernenden klargemacht, dass diese Sprache noch präsent ist, wie Dialekte im eigenen Land und in der eigenen Kultur. Es kann auch dazu genutzt werden, verschiedene Dialekte der Lernenden anzusprechen und sogar, dass der Dialog bei McDonalds mit verschiedenen Dialekten des eigenen Landes mehrfach gespielt wird. Ein anderes, tiefgründigeres Thema dieses Witzes ist auch die Unterstellung, dass Österreicher kein Englisch verstehen, da das „,chicken“" als ,schicken“ wahrgenommen wird.

Dieser Witz scheint sehr geeignet für den Einsatz im Fremdsprachenunterricht zu sein, da die Situation im McDonalds fast Jedem/ Jeder bekannt ist. Der letzte Satz ist allen Rezipienten bekannt, denn man hat in einem Restaurant nur zwei Möglichkeiten „Mitnehmen oder Hier-Essen“. Da er sprachlich auch nicht als schwierig einzustufen ist, kann dieser Witz im Fremdsprachenunterricht benutzt werden, um ein Phänomen der deutschen Sprache und die Unterschiede zwischen den Ländern Österreich, Deutschland und dem eigenen Land klarzustellen. Im Gemeinsamen Europäischen Referenzrahmen für Sprachen (2001) wird diese Fertigkeit als „Transaktionen: Dienstleistungsgespräche“ unter dem Niveau A2 angegeben (vgl. Europarat 2001:84). Am Ende des Themas ist wünschenswert, dass bei den Lernenden ein Bewusstsein für den österreichischen Dialekt, für den Unterschied zwischen Deutschland und Österreich und den Dialekten im eigenen Land entsteht. Für die zukunftsbezogene Perspektive für Deutschlernende, ist das Bewusstsein darüber, dass es Dialekte des Deutschen gibt und man auf sie zutreffen kann. Dieser Unterrichtsvorschlag ist auch exemplarisch nach dem Modell didaktische Analyse, da in jedem Lehrbuch für Deutsch zumindest der österreichische Dialekt thematisiert wird. Dieses kann als Vertiefung genutzt werden. Diese Didaktisierung entspricht demnach den Kriterien ,äußere Gestalt“ durch zufügen von Logos zur Vorwissensaktivierung und ebenfalls dem Kriterium „Adressaten der Texte", wobei sie zum Beispiel aufgefordert werden eigene Rollenspiele vorzutragen. Außerdem enthält der Unterricht eine Beschäftigung mit sprachlicher Vielfalt in diesem Fall das österreichische Deutsch, welches unter dem ,,phonetischen Bereich“ Çakırs (2006) als „dialektale Vielfalt“" dargelegt wird. 


\subsubsection{Witz, 2: Wahrnehmung der türkischen Sprache}

„Neulich beim türkischen Glücksrad. Kandidat: ,Ich kaufe ein Ü! ‘.

DING-DING-DING-DING-DING-DING-DING-DING-DING-DING-DING-DING ...“ (http-6).

Dieser Witz ist kultur- und sprachspezifisch, es macht die türkische Sprache zum Gegenstand. So wird die türkische Sprache nämlich in Deutschland wahrgenommen „Eine Sprache voller „Ü“s. Die Pointe dieses Witzes ist das „DING DING DING...", was besagt, dass sehr viele Buchstaben an der Glücksradtafel leuchten. Den Türken außerhalb Deutschlands ist diese Wahrnehmung wahrscheinlich nicht bekannt. Der Laut „Ü“ mit dem selben Graphem und derselben Aussprache existiert wahrscheinlich in wenigen Sprachen. Das Deutsche und das Türkische haben dieses Phänomen gemeinsam. Somit ist dieser sprachbezogene Witz für türkische Lernende sprachlich zu verstehen. Dass der Witz die türkische Sprache zum Gegenstand macht, entspricht der gegenwartsbezogenen Perspektive nach dem Modell „didaktische Analyse“. Die Frage der Zugänglichkeit für Lernende wird im Weiteren beschrieben.

Für den Unterricht kann folgendermaßen vorgegangen werden, dass man zum Einstieg den Lernenden fragt „Wie klingt Deutsch für Sie?“. Aufgrund der Eigenschaften der deutschen Sprache wie z.B. dass viele Konsonanten hintereinander stehen können, das für türkische Muttersprachler eine Herausforderung sein kann oder aber die Assoziation des Deutschen im historisch-militärischen Kontext, nehmen Menschen mit anderen Muttersprachen die deutsche Sprache als eine „hartklingenden Sprache“ wahr (vgl. Stickel 2019: 4f.). Nach dem Sammeln einiger Adjektive kann die Frage folgen „Und was glauben Sie, wie Türkisch für Deutsche klingt“. Kaum ein Muttersprachler stellt sich wohl jemals diese Frage, deshalb kann dieses etwas Zeit zum Nachdenken beanspruchen, einige Diskussionen innerhalb von Gruppen werden notwendig sein. Auf die Frage, wie Türkisch von Deutschen wahrgenommen wird, kann nun der Witz präsentiert werden mit der Erklärung des Glücksrads, dessen türkische Entsprechung existiert. Es wäre vorteilhafter, wenn die Lehrkraft den Witz vorliest mit der Betonung der am Ende leuchtenden Kästchen, die durch das „ய̈“ aufgehen. Dieses wird die Lernenden wahrscheinlich überraschen, da sie kein solches Bild über ihre eigene Sprache haben. Nun kann darüber gesprochen werden, inwieweit dieses Phänomen für die türkische Sprache zustimmt. Dieses kann für ein Sprachbewusstsein dienen, indem man anspricht, was die türkische Sprache von anderen Weltsprachen unterscheidet, was die deutsche Sprache von anderen Sprachen unterscheidet u.Ä. Am Ende des Unterrichts wäre es wünschenswert, dass ein Bewusstsein dafür entsteht, dass solche Wahrnehmungen subjektiv sind. Falls von der Klasse vorurteilshafte Wahrnehmungen zur deutschen Sprache existieren, wie es in anderen Ländern der Fall ist (vgl. Stickel 2019) könnten diese durch den Vergleich mit der Muttersprache bewusst gemacht werden, sodass sie sich zumindest etwas mindern. Somit dient dieser Witz auch zur Thematisierung eines in Deutschland vorherrschenden Stereotyps. Damit soll sie Frage der zukunftsbezogenen Perspektive beantwortet werden. Die Lernenden sollen hier ihre eigene Meinung über die Wahrnehmung zur deutschen Sprache äußern, 
dabei liegt der sprachliche Fokus auf der Meinungsäußerung. Der gemeinsame europäische Referenzrahmen gibt diese Fertigkeit für das Niveau A2 an. Für detailliertere Begründungen der Meinung ist mindestens das Niveau B1 notwendig (vgl. Europarat 2001: 82). Der Unterrichtsvorschlag ist exemplarisch für Deutschlernende in der Türkei, da es sich um die türkische Sprache handelt.

\subsubsection{Witz 3: So spricht ein Türke Deutsch}

„Ein Türke in einem Beerdigungsinstitut: - Guten Tag! Brauchen Grabstein. Drauf schreiben: ,Ali tot. '.

- ,Guter Mann, bei uns ist es üblich, dass man ein paar Worte mehr auf den Grabstein schreibt!‘

- ,Is gut: Ali tot, verkaufen Ford Transit!‘ (http-7).

Dieser Witz ist insofern ein sprachbezogener Witz, da es sich um die Sprachbarriere zwischen den Protagonisten handelt. Ein „Türke“ befindet sich in einem Beerdigungsinstitut und macht mit seinem ,unvollständigen“ Satzbau verständlich, dass er ein Grabstein für seinen verstorbenen Freund Ali mit der Aufschrift „Ali tot“" bestellen möchte. Daraufhin klärt ihn der Angestellte über den deutschen Brauch auf, dass man einige weitere Worte auf den Grabstein schreibt, woraufhin der „Türke“ dies nicht als Worte über den Verstorbenen versteht, sondern als eine weitere Mitteilung oder Bekanntmachung, sodass er einen für Inserate typischen Text diktiert. Er hat nämlich einen Wagen zu verkaufen. Diese Stelle ist die Pointe des Witzes. Der Witz beinhaltet einige Stereotype. Erstens, das Stereotyp, dass der Türke kein richtiges Deutsch spricht und zweitens, dass er so gefühlskalt ist, dass er eine Autoanzeige auf einen Grabstein von einem Bekannten aufgeben kann. Weitere Eigenschaften können natürlich hinzugefügt werden, wobei sie alle negativer Natur sein werden, da es als nicht angemessen angenommen wird so etwas auf einen Grabstein schreiben zu wollen. Es handelt sich hierbei zwar um einen Türken, diese Nationalität kann aber beliebig ausgetauscht werden, da diese Eigenschaft nicht nur einer Gruppe zugeschrieben werden kann.

Im Unterricht ist es sinnvoll für diesen Witz das Thema „Türken in Deutschland“" aufzugreifen. Viele Türken haben Verwandte, die als Gastarbeiter in den 1960er Jahren nach Deutschland ausgewandert sind. Einige haben also eine Vorstellung über die Situation. Nun kann als Anregung die Frage „Welche Probleme kann man als Ausländer in einem neuen Land haben?“ dienen. Das Sprachproblem wird zweifellos eines der ersten Bemerkungen sein. Somit kann der Gegenwartsbezug hergestellt werden, da man Migration auch in der Türkei kennt. Somit schafft man ebenfalls den Grund für die Zugänglichkeit zum Thema.

Mit dem Bewusstsein der Sprachbarriere kann nun der Witz präsentiert werden, mit den Ergänzungen, was ein Beerdigungsinstitut und ein Grabstein sind. Den Lernenden sollte das „Deutsch“ des „Türken“ auffallen. Sie werden vielleicht nicht lachen, aber sie werden den Witz verstehen, da es auch in der türkischen Kultur üblich 
ist, einige Worte über die Verstorbenen auf den Grabsteinen festzuhalten und dass dieses „Inserat“" nicht dazugehört.

Dieser Witz ist auch insoweit interessant, dass man während der Recherche auf denselben Witz auf Türkisch stoßen kann, nur nicht über einen „Ausländer“, sondern über die Menschen aus der Stadt Kayseri:

\begin{abstract}
Kayserili'nin eşi ölmüş, yerel bir gazeteye duyuru ilanı vermeye gitmiş. En ucuzundan standart bir ilan vermek istemiş. Önüne konan kağıda istediği ilanı yazmış: "Ayşse'yi kaybettim, üzgünüm”. İlan görevlisi ilanı görünce uyarmış: "İsterseniz 6 kelimeye kadar uzatabilirsiniz, üç kelime daha hakkınız var". Kayserili, "aynı paraya mı?" demiş. Görevli, "evet aynı paraya" deyince Kayserili üç kelime daha eklemiş: "Satılık Toyota var.(http-8)
\end{abstract}

In dem Witz geht ein Mensch aus Kayseri zum Beerdigungsinstitut und möchte einen Grabstein für seine verstorbene Frau fertigen lassen, woraufhin derselbe Dialog stattfindet und er am Ende hinzufügen lässt „Toyota zu verkaufen“.

Es ist eine tolle Gelegenheit, die auf jeden Fall im Unterricht benutzt werden sollte, da somit klar wird, dass diese Art Witze nicht die Realität wiederspiegeln. Es kann davon ausgegangen werden, dass Türken außerhalb Kayseri über diesen Witz lachen werden, da Menschen aus Kayseri allgemein im Rest des Landes als ,gerissen“ angenommen werden, im Sinne von ,auf seinen Vorteil bedacht“ ganz besonders in Geldangelegenheiten und im Handeln. Dies kommt eigentlich davon, dass in der Geschichte von Kayseri viele unterschiedliche Zivilisationen diesen Ort als Handelszentrum nutzen und somit diese Fertigkeit immer weiterentwickelt wurde. Wenn nun aber erst der Witz über den Türken bearbeitet wird und dann der über Kayseri, wird der Zweite wahrscheinlich nicht nur noch als komischer Witz aufgefasst. Es wird erwünscht, dass er als Stereotyp angesehen wird, das ist der zukunftsbezogene Aspekt des Vorschlags. In dem Witz gibt es auch kurze, unvollständige Sätze, da es sich um gesprochene Sprache handelt. Dieses findet man auf diese Art nicht in Lehrbüchern. Der Witz beinhaltet somit einige Kriterien Cakirs (2006) auf die ,sprachliche Vielfalt“ bezogen im ,syntaktischen, diskursiven Bereich“. Dieser Witz unterstreicht außerdem den Aspekt, den Schu / Schu in ihrer Arbeit darlegen. Hier ist der ethnische bzw. nationale Stereotyp nicht konstitutiv, das heißt die Ethnie oder die Nationalität im Witz kann ausgetauscht werden und die Pointe des Witzes bleibt beibehalten. Dieser Aspekt dient der Frage, dass der Unterricht exemplarisch ist. Dieser Unterricht hat eine etwas detailliertere Beschäftigung mit der Migrationsgeschichte der Türken nach Deutschland und die Präsentation der Ergebnisse, außerdem kann über die aktuelle Situation im eigenen Land gesprochen werden dafür sieht der Referenzrahmen das Niveau B1/ B2 vor (Europarat 2001: 66).

\title{
3.2.4 Witz 4: Wahrnehmung der französischen Sprache
}

„Treffen sich zwei Freundinnen im Café und unterhalten sich über ihre Freizeitaktivitäten. Erzählt die eine: ,Ich lerne jetzt Französisch!‘ ,Mach das nicht‘, 
antwortet darauf die andere, ,ich kenne eine, die wäre bei der mündlichen Prüfung beinahe erstickt!‘ (http-9).

Dieser Witz behandelt die französische Sprache und ihre Wahrnehmung in der deutschen Gesellschaft. Zwei Freundinnen unterhalten sich über ihre Freizeit, wobei eine davon erzählt, dass sie angefangen hat Französisch zu lernen. Die andere ratet ihr davon $a b$, weil eine andere Freundin, aufgrund einiger Besonderheiten bei der Aussprache, wie die Nasalen, in der mündlichen Prüfung „,beinahe erstickt" wäre. Somit enthält dieser sprachenbezogene Witz einen negativen Stereotyp über die französische Sprache.

Im Unterricht kann die Einstiegsfrage „Wie klingt die französische Sprache für Sie?" begonnen werden. In der Türkei wird die französische Sprache von Schüler / innen meist als „romantisch“ beschrieben (vgl. Alpar / Kartal 2019: 413; Arslan 2021: 65). Hier wird ein Unterschied zur deutschen Wahrnehmung im Witz entstehen. Nachdem Meinungen zum Klang der Sprache gesammelt werden, kann der Witz präsentiert werden. Somit wird der Gegenwartsbezug hergestellt. Zur Zugänglichkeit tragen der oben beschriebene Teil und die folgenden Schritte bei.

Aus sprachlichen Gründen ist der Witz nicht sehr schwer zu verstehen, bis auf das Wort „erstickt“, das geklärt oder den Lernenden selbst zum Nachschauen überlassen werden kann. Wahrscheinlich werden die Lernenden den Grund für das Ersticken nicht erkennen, da wie gesagt, der türkische Blick auf die Sprache eine andere ist. Dieses sorgt also dafür, zu erkennen, dass dieses ein Stereotyp ist, der nicht der Wahrheit entspricht. Dieses ist für Zukunftsbezogenheit von Bedeutung. Außerdem enthält der Witz das Grammatikthema „Konjunktiv II“ in der Vergangenheit. Dieses kann hier wiederholt werden, sollte schon vorher behandelt worden sein.

Es kann zum Vergleich mit dem Blick der Türken auf die deutsche Sprache verglichen werden und so erreicht werden, dass auch dieses eine subjektive Wahrnehmung ist. Der Witz kann für einen Vergleich in jedem anderen Land genutzt werden, da Französisch eine weitverbreitete Fremdsprache ist, können viele eine Meinung über die Sprache haben, somit wird dieser Vorschlag als exemplarisch vorgesehen. Da der Witz grammatisch das Thema Konjunktiv II aufweist, empfiehlt es sich hierbei vom Niveau B1 nach dem Referenzrahmen auszugehen (Europarat 2001: 216).

\subsubsection{Witz 5: Bayrisch}

„Ein Bayer hat sich verirrt und fragt einen Türken, der des Weges kommt: ,Entschuldigung, wo geht‘s denn do noch Aldi?‘. Der Türke verbessert: ,Zu Aldi‘“،.

Darauf der Bayer: ,Ach, Aldi hot scho zua?‘ (http-10).

Dieser Witz scheint sich sehr gut für den Einsatz im Unterricht zu eignen, da er viele Anregungen $\mathrm{zu}$ verschiedenen Themenbereichen des Fremdsprachenunterrichts gibt. Ersteres ist die der Kultur und Landeskunde. Um diesen Witz, der sich an 
Stereotypen bedient, zu verstehen muss man wissen, dass es einen bayerischen Dialekt in Deutschland gibt, der auch heute noch sehr präsent im Bundesland ist. Ein Bayer fragt einen Türken nach dem Weg zu Aldi. Der Bayer spricht seinem Dialekt nach richtig und benutzt, ein, der Präposition „nach“ im Hochdeutschen ähnliches, Wort nämlich das „,noch“, der Türke verbessert seinerseits die Präposition, auf Hochdeutsch von seinerseits „,nach“ zur korrekten Präposition „zu“. Da der Bayer nicht auf die Idee kommt, dass der Türke ihn grammatisch verbessert, versteht er ihn falsch und interpretiert das ,zu“ als das Präfix ,zu“ von ,zuhaben“, im Sinne von geschlossen sein. Somit ist dieser ein lexikalischer Witz.

Die Beschäftigung für Lerner mit Witzen, in denen die eigene Kultur vorkommt, wird mit Sicherheit das Interesse erwecken. Hier bekommen die Lernenden einen Eindruck darüber, wie Türken in Deutschland angesehen werden. Dieses ist ein sehr umfangreiches Thema, welches eine gemeinsame Geschichte der beiden Länder beinhaltet. Dieser Aspekt spricht für den Gegenwartsbezug der Lernenden, außerdem kann der Vorschlag somit als exemplarisch für die Deutschlernenden in der Türkei gelten.

Vor der Arbeit mit dem Witz wäre es demnach sinnvoll auf diese Zeit einzugehen. Am einfachsten könnten Lernende Präsentationen $\mathrm{zu}$ den jeweiligen Themen der Migration vorbereiten, sodass ein Rahmen entsteht. Nach den Präsentationen sollte sich herausstellen, dass die Türken nun seit Jahrzehnten in Deutschland leben und dass typische Bilder von Deutschen über Türken und umgekehrt existieren. Der Zugang zum Thema sollte hiermit vorbereitet werden. Dieses sollte als Übergang zum Thema „Aldi“ genutzt werden. Schließlich bedeutet „Aldi“ einem Lernenden, der sich noch nie in einem deutschsprachigen Land befand, nichts. Hierfür sollte „Aldi“ als Supermarktkette vorgestellt werden. Zum Beispiel kann das Logo projiziert werden, sodass die Lernenden raten sollen, worum es sich dabei handelt. Der Witz wurde bis jetzt noch nicht präsentiert, aber dennoch wurden schon sehr viele landeskundliche Elemente von vornehinein im Unterricht behandelt, da diese das Verständnis erleichtern. Nachdem der Supermarkt als solches identifiziert wurde, sollte die Klasse sich mit der Frage des Zusammenhangs zwischen Türken und Aldi beschäftigen. Es kann vielleicht vermutet werden, dass die meisten Türken hier arbeiteten oder noch arbeiten, dass sie vielleicht die Gründer von Aldi sind mit der Anspielung auf das türkische Wort „aldı-(satın) almak“ im Sinne von „gekauft-kaufen“, vielleicht taucht auch die Vermutung auf, dass Türken viel bei dem Supermarkt einkaufen. Mit dieser Vermutung könnten einige Bilder von, besonders kopftuchtragenden, türkischen Frauen mit der „Aldi-Tüte“ in der Hand präsentiert werden.

Jetzt kann der Witz der Klasse präsentiert werden. Es ist zu erwarten, dass sie die bayrischen Textstellen nicht verstehen werden, deshalb sollten sie vor die Aufgabe gestellt werden, was das bedeuten kann. Dafür müssen die Lernenden mit der Tatsache bekannt gemacht werden, dass in Deutschland verschiedene Dialekte gesprochen werden. Im allgegenwärtigen DaF-Unterricht und in den Lehrwerken findet man meistens Beispiele zum Deutsch in Österreich oder in der Schweiz, die oberflächlich 
Unterschiede präsentieren. Dieses bietet wiederum die Möglichkeit sich detaillierter mit Dialekten des Deutschen zu beschäftigen.

Es bietet sich die Gelegenheit an den Lernenden die Entschlüsselung dieser Zeilen als beispielsweise Hausaufgabe aufzugeben. Hierbei wird „nur“ beabsichtigt, dass man sich mit dem Dialekt etwas näher auseinandersetzt, da besonders der bayrische Dialekt sehr stark präsent in Deutschland ist. Nun müssen also die bayrischen Textstellen geklärt werden. Dadurch wird man auf das Thema der Präpositionen ,zunach“ und „dem Präfix ,zu“ aufmerksam.

Ein Türke verbessert also einen Bayer. Es sollen nun Überlegungen gemacht werden, was das zu bedeuten hat. „Ein Türke spricht besser Deutsch als ein Deutscher“, da er die Präposition verbessert. Es sollte auffallen, dass es in Deutschland ein stereotypisches Bild eines Bayers existiert, der das Standarddeutsche missverstehen kann und dass es witzig ist, dass ihn ein Türke verbessert. Dieses bietet den zukunftsbezogenen Aspekt nach dem Modell „didaktische Analyse“ Dieser Unterricht hat eine etwas detailliertere Beschäftigung mit der Migrationsgeschichte der Türken nach Deutschland und die Präsentation der Ergebnisse, dafür sieht der Referenzrahmen das Niveau B1/ B2 vor (Europarat 2001: 66).

\section{Schlussfolgerung}

In dieser Arbeit wurde versucht aufzuzeigen wie sprachbezogene Witze als authentische Materialien im Fremdsprachenunterricht ihren Platz finden können. Der Einsatz von authentischen Materialien im Unterricht wird seit der kommunikativen Wende befürwortet, um unter Anderem, den pragmatischen Sprachgebrauch in den Unterricht besser zu integrieren. Sprachbezogene Witze können dabei Stereotype über bestimmte Gruppen enthalten und dieses stellt für die Lehrkraft eine besondere Herausforderung dar. Es geht bei sprachbezogenen Witzen nämlich nicht vorrangig um die Vermittlung sprachlicher Aspekte, wie zum Beispiel die Grammatik. Bei der Erstellung der Vorschläge wurden die Leitfragen des Modells „didaktische Analyse“, nach Klafki (zitiert nach Bimmel 2011), beachtet. Als vorrangiges Ziel beim Einsatz von sprachbezogenen Witzen haben wir uns die Vermittlung von Landeskunde und die bewusste Arbeit mit Stereotypen gesetzt. Somit spielen in solchen Unterrichtsreihen besondere Gefühle eine Rolle. Die Gruppe kann sich gekränkt fühlen oder auch amüsieren. Damit gilt es zu arbeiten. Durch die Didaktisierung der ausgewählten sprachbezogenen Witze wurde versucht Wege zu zeigen, wie man Stereotype im Unterricht bewusst wahrnehmen kann. Besonders durch den Vergleich mit Witzen oder ähnlichen Stereotypen aus der eigenen Kultur können Lernende erkennen, dass es sich um Stereotype handelt und nicht unbedingt der Realität entspricht. Diese Wahrnehmung trägt zur Ausbildung einer interkulturellen Kompetenz bei, dass als eines der Ziele des Fremdsprachenunterrichts beschrieben wird. Im gemeinsamen europäischen Referenzrahmen für Sprachen wird der bewusste Umgang mit Stereotypen als Bestandteil der interkulturellen Kompetenz beschrieben (Europarat 2001: 106). Es wurde in der Arbeit versucht aufzuzeigen, dass sprachbezogene Witze hierzu einen 
großen Beitrag leisten können. Die bewusste Wahl, besonders der Witze in denen es um die eigene Sprache und Kultur geht und die bewusste Auseinandersetzung mit diesem Thema ist von besonderer Bedeutung und sollte nicht außer Acht gelassen werden, da ohne Aufklärung bei Lernenden, vor allem durch das Internet, Missverständnisse aufkommen können. Der gemeinsame europäische Referenzrahmen beschreibt folgende Fertigkeiten für die interkulturelle Kompetenz

\begin{abstract}
Die Fähigkeit, die Ausgangskultur und die fremde Kultur miteinander in Beziehung zu setzen; - Kulturelle Sensibilität und die Fähigkeit, eine Reihe verschiedener Strategien für den Kontakt mit Angehörigen anderer Kulturen zu identifizieren und zu verwenden; - Die Fähigkeit, als kulturelle Mittler zwischen der eigenen und der fremden Kultur zu agieren und wirksam mit interkulturellen Missverständnissen und Konfliktsituationen umzugehen; - Die Fähigkeit, stereotype Beziehungen zu überwinden. (Europarat 2001: 106)
\end{abstract}

Mit den vorgeschlagenen Aktivitäten für die Unterrichtseinheiten wurde versucht darzulegen, dass sprachbezogene Witze für die Entwicklung dieser Fertigkeiten geeignet sind. Im Besonderen wurde Witz 5 dazu genutzt ein tieferes landeskundliches Wissen zu vermitteln, welches in Lehrbüchern in verschiedenen Formen vorzufinden ist. Somit wurde beabsichtigt ein neues Bild über die Zielkultur zu präsentieren, sodass ein Vergleich mit der eigenen Kultur getätigt werden kann. Witz 2, 3 und 4 spiegeln dagegen eher Stereotype der Zielkultur. Diese sind manchmal gegen die eigene Kultur und manchmal über andere Kulturen. In den ersteren Fällen wird ein Blick darüber geschaffen, wie man selbst in einer anderen Kultur dasteht. Durch die detaillierte Beschäftigung mit dem Stereotyp und dem Witz wird aber eine Stufe erreicht, die erkennen lässt, dass dieses Bild nicht der Realität entspricht, sondern eben einen Stereotyp wiedergibt, der auch in der eigenen Sprache über andere existiert. Letzteres dagegen regt sofort zum Vergleich mit der Wahrnehmung einer Sprache oder Kultur im eigenen Land an. Somit erkennt man, dass solche Wahrnehmungen von Land zu Land, von Kultur zu Kultur variieren. Es ist wünschenswert, dass diese Erkenntnis wiederum $\mathrm{zu}$ einer interkulturellen Kompetenz und zur Sensibilisierung für Stereotype und Vorurteile führt. Dieses kann als Antwort zur ersten Forschungsfrage dienen Diese Erkenntnis führt wiederum zu einer interkulturellen Kompetenz. Dieses kann als Antwort zur ersten Forschungsfrage dienen.

Zur zweiten Forschungsfrage kann festgehalten werden, dass für den Einsatz der Witze im Unterricht es eine Voraussetzung ist, dass die Lehrkraft über das notwendige Wissen verfügt. Dieses ist zweifelsohne ein wesentlicher Aspekt, wenn es darum geht, Witze zu behandeln Sowohl die Lehrkraft als auch die Lernenden müssen in der Lage sein die Witze zu verstehen. Konkret für die Witze in unserer Arbeit kann festgehalten werden, dass für das Verstehen des Witzes 1 sind vorerst sprachliche Voraussetzungen zu erfüllen. Da es sich um einen Dialog handelt, bei dem etwas bestellt wird, sollte den Lernenden die Konstruktion „Ich hätte gern...“ bekannt sein. Des Weiteren sollte ihnen bekannt sein, dass man in Österreich einen Dialekt des Standarddeutschen benutzt, dass „Hendl“ „Hähnchen“ im Standarddeutschen bedeutet wird zwar im Laufe des Dialogs klar, dennoch erleichtert es das Verstehen der Lernenden, da sie schon durch das österreichische Deutsch verwirrt sein werden. Für das Verstehen von Witz 2 ist das 
Kennen der Sendung Glücksrad notwendig. Witz 3 dagegen erfordert kein Vorwissen, außer vielleicht den Worten Beerdigungsinstitut und Grabstein. Für Witz 4 muss man über den Klang der französischen Sprache Bescheid wissen. Witz 5 erfordert wiederum ein historisches Wissen über die türkischen Gastarbeiter in Deutschland, den bayrischen Dialekt und den Präpositionen und Präfixen. Außerdem braucht man die Information, dass „Aldi“ eine Supermarktkette in Deutschland ist. Zusammenfassend lässt sich sagen, dass bestimmte sprachliche Voraussetzungen notwendig sind, aber auch ein Weltwissen und landeskundliches Wissen notwendig sind, um mit dieser Art von Witzen umzugehen. Hierbei ist es wichtig, dass die Lehrkraft über diese ohne Zweifel zu verfügen hat. Den Lernenden diese zu vermitteln kann aber eines der Lernziele des Unterrichts sein. Grammatische Themen mit sprachbezogenen Witzen zu vermitteln scheint nicht geeignet zu sein, dafür aber bieten diese Art Witze und mit Sicherheit auch andere Arten, neue Wörter kennenzulernen. Als übergeordnetes Lernziel jedoch tritt die interkulturelle Kompetenz zum Vorschein, ganz besonders in Bezug auf die Arbeit mit Stereotypen und landeskundlichem Wissen. Eine Studie mit der Anwendung der Witze dieser Arbeit und Witzen aus anderen Kategorien könnte aufzeigen, welche Art von Witzen türkischen Deutschlernenden schwerer oder leichter fällt. Dazu könnte auch die Arbeit von Semiz (2014) eine Orientierung bieten, denn demnach könnte man die Witze im Unterricht für den weiteren Verlauf beurteilen, worauf Hargaßner (2018) ebenfalls aufmerksam macht.

Lehrkräfte, die die Zielsprache ebenfalls als Fremdsprache erlernt haben und somit die Zielkultur ebenfalls nur aus Büchern oder anderen Medien kennen, werden vor die Aufgabe gestellt sich tiefer mit dem Hintergrund eines Themas in einem Witz zu beschäftigen, da ihnen im Vergleich zu Muttersprachlern dieses bestimme Wissen fehlen wird. Wenn man nun davon ausgeht, dass der Großteil der Lehrkräfte einer Fremdsprache, keine Muttersprachler sind, kann es von Vorteil sein in den jeweiligen Abteilungen der Universitäten, während der Lehrerausbildung, ein entsprechendes Programm zu „sprachbezogenen“ Witzen anzubieten. In der Türkei sind Lehrkräfte des Deutschen als Fremdsprache größtenteils ebenfalls Fremdsprachler, dies kann man daraus schließen, dass dreizehn der sechzehn Abteilungen für Deutschlehrerausbildung in der Türkei ihre Studierenden mit einer Englischsprachprüfung aufnehmen und ihnen ein obligatorisches Vorbereitungsjahr für die deutsche Sprache anbieten (vgl. ÖSYM 2020: 159 ff.). Dass, das Profil der Deutschlehrkräfte in der Türkei sich von Muttersprachlern zu Fremdsprachlern wandelt bestätigt auch die Arbeit von Şahin und Öztürk (2020). Witze als Träger von Stereotypen und Landeskunde und ihr Einsatz im Fremdsprachenunterricht kann auch als Fortbildungsprogramm durch beispielsweise das Goethe Institut oder das Kultusministerium angeboten werden. Es ist wünschenswert, dass diese Fortbildungen dazu führen, dass Lehrkräfte das Angebot an Witzen aus der Zielkultur vermehrt in ihren Unterricht integrieren. Außerdem wäre es auch interessant zu sehen, wie Deutschlernende auf diese Art Witze reagieren und welche Art von Witzen für sie leichter zu verstehen ist. Somit könnte man vielleicht eine Sammlung für den DaF-Unterricht, insbesondere in der Türkei erstellen, das sprachbezogene Witze und Witze im Allgemeinen enthält, mit einem Lehrerhandbuch, das Lehrkräften eine 
Orientierung bietet. Sprachbezogene Witze insbesondere, eignen sich nach den Überlegungen dieser Arbeit, als Einstieg in das Thema zum bewussten Umgang mit Stereotypen, sollten aber nicht als einziges Mittel herangezogen werden. $\mathrm{Zu}$ Forschungsfrage drei, wie konkreter Unterricht mit „sprachbezogenen Witzen“ gestaltet werden kann, wurde versucht mit den Didaktisierungsvorschlägen eine Antwort zu geben. Zusammenfassend lässt sich hier festhalten, dass auf „sprachebezogene Witze“ nach dem Modell „didaktische Analyse“ auf unter anderem die Gegenwart und Zukunft der Lernenden bezogen werden kann. Außerdem wurden konkrete Vorschläge zum interkulturellen Vergleich angeboten, sodass diese im Unterricht angewendet werden können.

\section{Literaturverzeichnis}

Ağaçsapan, Asuman / Ünal, Bilge / Balkaya, Şengül (2018): Witze als interkulturelle Vermittlung in der DaF-Didaktik. In: Anadolu University Journal of Education Faculty, (Special Issue), 15-24.

Alpar, Melek / Kartal, Erdoğan (2019): Fransızcanın rengi: Öğretmen adaylarının yabancı dil olarak Fransızcaya dair metaforik algıları. In: RumeliDE Dil ve Edebiyat Dergisi. 2019. Say1 17, 405-416.

Arslan, Nahide (2021): “6. ve 7. Sınıf Öğrenclerinin Fransızca Öğretimine İlişkin Metaforik Algıları. In: Karamanoğlu Mehmetbey Ünversitesi Uluslararası Filoloji ve Çeviribiliim Dergisi. 3(1), 46-71.

Auswärtiges Amt (2020): Deutsch als Fremdsprache weltweit. Datenerhebung 2020, 26-27. https://www.goethe.de/resources/files/pdf204/bro_deutsch-als-fremdsprache-weltweit.datenerhebung-2020.pdf (Letzter Zugriff: 11.10.2021).

Bimmel, Peter / Kast, Bernd / Neuner, Gerhard (2011): Deutschunterricht planen neu. Kassel: Langenscheidt.

Çakır, Gülcan (2006): Zur Frage der Authentizität in Lehrwerken des Deutschen als Fremdsprache. Hamburg: Dr. Kovac Verlag.

Europarat für kulturelle Zusammenarbeit (Hg.) (2001): Gemeinsamer Europäischer Referenzrahmen für Sprachen: Lernen, Lehren, Beurteilen. Berlin, München: Langenscheidt. Online- Version: https://www.goethe.de/z/50/commeuro/i0.htm (Letzter Zugriff 25.04.2021).

Gaudo, Felix / Kaiser, Marion (2018): Lachend lernen: Humortechniken für den Unterricht. Weinheim: Julius Beltz.

Hargaßner, Julia (2018): Perspektiven der Witzforschung im Kontext des Russischunterrichts. Didaktik der slawischen Sprachen - Beiträge zum 1. Arbeitskreis in Berlin (12.-14.9.2016). Innsbruck: University Press.

Hascher, Tina / Edlinger, Heidrun (2008): Von der Stimmungs- zur Unterrichtsforschung: Überlegungen zur Wirkung von Emotionen auf schulisches Lernen und Leisten. In: Unterrichtswissenschaft. 36(1), 55-70.

Herrmann, Christoph / Fiebach, Christian (2007): Gehirn und Sprache: Funktionelle Bildgebung, neurokognitive Modelle, Sprachareale, Sprachstörungen, Sprachevolution, Sprachgene, Dyslexie, Lateralisierung, Sprache und Emotionen, Mehrsprachigkeit, Gebärdensprache. Frankfurt: Fischer Taschenbuch.

Hoffmann, Magdalena (2010): Die Mehrdeutigkeit als Träger des sprachlichen Witzes. In: Acta Universitatis Lodziensis. Folia Germanica. 6, 79-94. 
Hohenhaus, Peter (2000): Zur Verwendung humoristischen Materials im DaF-Unterricht. In: Informationen Deutsch als Fremdsprache. 27(4), 433-448.

Huneke, Hans Werner / Steinig, Wolfgang (2000): Deutsch als Fremdsprache: Eine Einführung. Schmidt, Erich.

Kotthoff, Helga (2006): Scherzkommunikation: Beiträge aus der empirischen Gesprächsforschung. Göttingen: Verlag für Gesprächsforschung.

Löschmann, Martin (Hg.) (2015): Humor Im Fremdsprachenunterricht. Frankfurt: Peter Lang Gmbh, Internationaler Verlag der Wissenschaften.

Mayer, Bernt / Brückner, Sarah (2011): Lebenslanges Lernen auf Basis Neurowissenschaftlicher Erkenntnisse: Schlussfolgerungen für Didaktik und Personalentwicklung. Weidener Diskussionspapiere, (29) (ohne Seitenangabe).

Middeke, Annegret / Murdsheva, Stanka (2008): Nationen- und Ethnowitze im interkulturellen DaF Unterricht. Humor: Grenzüberschreitende Spielarten eines kulturellen Phänomens. Göttingen: Universitätsverlag.

ÖSYM (2020): 2020 Yükseköğretim Kurumları Sinavı (Yks) Yükseköğretim Programları ve Kontenjanları Kılavuzu. Ankara: ÖSYM.

Roche, Jörg (2005): Interkulturalität. Daf-Didaktik Und Daf-Unterricht. In: Neuland, Eva / Ehlich, Konrad / Roggausch, Werner (Hg.): Perspektiven der Germanistik in Europa Tagungsbeiträge. München: Iudicium, 159-169.

Şahin, Hamdullah / Öztürk, Kadriye (2020): Almanca Öğretmenliği Programı Öğrencilerinin Bakış Açısından Çift Anadal Programları. In: Diyalog Interkulturelle Zeitschrift Für Germanistik. Sonderausgabe: 85 Jahre Germanistik in der Türkei, 92-124.

Schu, Josef / Schu, Ursula (1998): Ethnische Witze im Fremdsprachenunterricht. In: Kwartalnik Neofilologiczny XLII 3, 293-307.

Semiz, Öznur (2014): EFL learners' Understanding of Linguistic Ambiguity in Language-based Jokes. In: Journal of Narrative And Language Studies. 2(2), 1-11.

Stickel, Gerhard (2019): Deutsch von außen. Berlin/ Boston: De Gruyter.

Szczęk, Joanna (2006): Man kann seine Nachbarn nicht wählen: Deutsche Polenwitze als Träger der Stereotype. In: Studia Germanica Gedanensia. 14, 169-179.

Ueda, Yasunari (2013): Textsorte Witz und Karikatur als Material zum Sprachlernen: Linguistische Ansätze zum Philosophieren mit Kindern. Berlin: Lit Verlag.

Üstün, Bilal / Tanrıkulu, Lokman (2021): Almanca Öğretmenliği Hazırlık Sınıfı Öğrencilerinin Almanca Derslerine Yönelik Tutumlarının Farklı Değişkenlere Göre İncelenmesi. In: Fırat Üniversitesi Sosyal Bilimler Dergisi. 31(3), 1211-1220.

Utri, Reinhold (2012): Witztexte für den DaF-Unterricht - zu Didaktisierungsmöglichkeiten anhand von deutschen und polnischen Musikerwitzen. In: Studia Niemcoznawcze, 587-599.

Vester, Frederic (2001): Denken, Lernen, Vergessen: Was geht in unserem Kopf vor, wie lernt das Gehirn, und wann lässt es uns im Stich? München: dtv Verlagsgesellschaft.

Voltrová, Michaela (2018): Witze im Zentrum oder an der Peripherie des DaF-Unterrichts? Der Witz im Spracherwerbsprozess aus der pragmalinguistischen Perspektive. In: Janíková, Vera / Nálepová, Jana (Hg.): Zentrum und Peripherie: aus fremdsprachendidaktischer Sicht. Opava: Slezská Univerzita v Opavě, 185-194.

Wahrig-Burfeind, Renate (Hg.) (2011): Brockhaus, Wahrig, Wörterbuch der deutschen Sprache. 9. vollst. neu bearb. und aktualisierte Neuausg. Gütersloh: Wissenmedia. 


\section{Internetquellen}

„Witz“ auf Duden online. https://www.duden.de/rechtschreibung/Witz (Letzter Zugriff: 25.05.2021).

http-1: https://schlechtewitze.com/krone (Letzter Zugriff: 16.11.2021).

http-2: https://schlechtewitze.com/witze/5cd442f06bfd2124d46d24c8/was-heisst-ofen-auf-chinesisch (Letzter Zugriff: 16.11.2021).

http-3: https://www.spruchmonster.de/woraus-machen-die-deutschen-ihre-autos-aus-krupp-stahlworausmachen-die-schweden-ihre-autos-aus-schw (Letzter Zugriff: 16.11.2021).

http-4: https://schlechtewitze.com/kaugummi (Letzter Zugriff: 16.11.2021).

http-5: https://www.spitzenwitze.de/oesterreicher/11576/ (Letzter Zugriff: 16.11.2021).

http-6: https://vic.bg/Witze/neulich-beim-t\%C3\%BCrkischen-gl\%C3\%BCcksrad (Letzter Zugriff: 16.11.2021).

http-7: https://schlechtewitze.com/t\%C3\%BCrken (Letzter Zugriff: 16.11.2021).

http-8: https://www.komikfikralar.org/kayseri-linin-olum-ilani.html (Letzter Zugriff: 16.11.2021).

http-9: https://vic.bg/witze/franzosen-witze?p=4 (Letzter Zugriff: 16.11.2021).

http-10: https://schlechtewitze.com/t\%C3\%BCrken?skip=20 (Letzter Zugriff: 16.11.2021). 